\title{
Reinigungsbranche im Umbruch
}

\author{
Mit Einbußen von bis zu 50 Prozent beim Auftragseingang war 2020 für die Hersteller von \\ Reinigungsanlagen ein mehr oder weniger rotes Jahr. Inzwischen hat die Nachfrage wieder \\ angezogen, aber mit deutlichen Unterschieden in den verschiedenen Branchen und Märkten.
}

Eigentlich hatte 2020 für die meisten Anlagenhersteller vielversprechend begonnen. Doch dann kam Corona und die Wirtschaft stand zunächst einmal still. Dies führte dazu, dass bereits erteilte Aufträge zwar nicht storniert aber verschoben wurden. Nach einer gewissen Schockstarre im März und April kam ab Mitte des zweiten Quartals wieder Bewegung ins Geschäft allerdings nicht konstant, sondern bei den meisten Herstellern in Wellen. Bemerkbar gemacht hat sich bei verschiedenen Unternehmen auch, dass die Messe parts2clean nicht stattfinden konnte. Bringt der Branchentreff doch jedes Jahr zahlreiche gute Kontakte, aus denen sich im Nachgang einige Aufträge generieren lassen.

Schwer taten sich die Kunden der Anlagenbauer damit, Entscheidungen zu treffen und aus bereits verhandelten Projekten eine Bestellung werden zu lassen. Nicht selten wurde die Situation ausgenutzt, Preise nochmals zu verhandeln. Die Hersteller berichten auch von verschärften Preisverhandlungen bei den Aufträgen, die vergeben wurden. Die Zahl der Auftragsrückgänge lag 2020 im Vergleich zu 2019 daher zwischen 20 und $50 \%$.

\section{Nicht nur durch Corona}

Einigkeit besteht darüber, dass diese Einbußen nicht nur der Pandemie geschuldet sind. Denn begonnen hat das Dilemma bereits Mitte 2018 durch die Transformationsprozesse in der Automobilindustrie und ihrer Zulieferer. Die Branche sicherte einem großen Teil der Anlagenhersteller über viele Jahre gute Geschäfte. Die Äußerungen großer OEM, nicht mehr in die Weiterentwicklung von Verbrennungsmotore zu investieren, sorgt nun für Verunsicherung. Und das unabhängig davon, dass auch Hybrid-Fahrzeuge mit einem Verbrennermotor ausgestattet sind.

Anlagen für die Reinigung von Komponenten für Diesel- und Benzinmotoren werden zwar von Zulieferern noch angeschafft, es handelt sich dabei jedoch häufig um Ersatzinvestitionen. Der Fokus der Automobilindustrie liegt jetzt auf batteriebetriebenen Fahrzeugen, und hier wird auch investiert. Diese Entwicklung hat sich durch Corona noch beschleunigt. Die Anlagenhersteller erwarten, dass sich die Nachfrage nach entsprechenden Reinigungslösungen in den kommenden Monaten verstärken wird.

\section{Marktpotenziale anderer Branchen}

Mehr oder weniger stark fallen auch die Investitionen in anderen Branchen aus. So war zu erfahren, dass sich aus Industriebereichen wie Medizintechnik, Optik, Elektronikindustrie, Sensortechnik, HalbleiterZulieferbereich, Hydraulik und Pneumatik, Beschichtungsindustrie, additive Fertigung und Recycling die Anfrage- und Projekttätigkeit sowie die Auftragseingänge in eine erfreuliche Richtung bewegen. Dabei handelt es sich überwiegend um Anwendungen, bei denen hohe (feinst-)partikuläre und/oder filmische Sauberkeitsanforderungen zu erfüllen sind.

Dies wäre auch bei Produktionsequipment für die Herstellung medizinischer Schutzausrüstung sowie von Rohstoffen und Medikamenten wie Impfstoffen der Fall. Dadurch könnte sich auch für die Hersteller von Reinigungslösungen ein Marktpotenzial ergeben. Dafür wäre es allerdings erforderlich, dass die Politik die entsprechenden Rahmenbedingungen schafft.

\section{Beziehungspflege auf Distanz}

Unabhängig von zum Teil noch bestehenden Reisebeschränkungen ist es den Anlagenbauern in den letzten zwölf Monaten gelungen, ihre Exportgeschäfte wieder in Fahrt zu bringen beziehungsweise $\mathrm{zu}$ beschleunigen. Einige Hersteller verzeichnen aus China eine stark anziehende Nachfrage, während sich bei anderen Herstellern diese Tendenz dagegen eher auf einem niedrigen Level hält. Gründe für die
Investitionszurückhaltung werden unter anderem in der Initiative „Made in China 2025“ und auch in eventuellen heraufziehenden Handelskonflikten zwischen den USA und Europa auf der einen und China auf der anderen Seite gesehen.

Eine Region, in der in den kommenden Jahren aufgrund einer zunehmenden Etablierung von Hightech-Unternehmen ein wachsender Bedarf an hochwertigen Reinigungslösen entstehen könnte, sind die ASEAN-Länder. In den USA und zum Teil auch in Mexiko hatte die Krise für verschiedene Hersteller nahezu keine Auswirkungen und das Geschäft läuft weiterhin auf einem sehr hohen Niveau, andere berichten von einem komplett eingebrochenen Markt. Aus Südeuropa sind vereinzelt Auftragseingänge zu verzeichnen. Bestellungen und Projektanfragen aus den skandinavischen Ländern, Benelux und Polen haben dagegen wieder deutlich zugelegt. Auswirkungen des Brexits waren praktisch nicht spürbar.

\section{Knappheit bei Rohstoffen und Chips}

Ein Wertmutstropfen bei den wieder erstarkenden Vertriebsaktivitäten der Anlagenbauer sind die stark gestiegenen Preise für Rohstoffe wie Edelstahl und die sehr eingeschränkte Marktverfügbarkeit von Mikrochips. Dies macht nicht nur die Kalkulation schwierig, sondern sorgt auch für länger als nötige Lieferzeiten. Sie liegen bei Sonderanlagen momentan bei bis zu neun Monaten, Standardanlagen können die Anlagenbauer zum Teil ab Lager beziehungsweise in einem Zeitraum von rund drei Monaten liefern.

Die insgesamt anziehende Nachfrage sorgt in der Branche für eine verhalten optimistische Stimmung. Es wird jedoch damit gerechnet, dass es einige Zeit dauern wird bis das Vor-Corona-Niveau wieder erreicht ist und auch, dass dafür noch einige Hürden zu nehmen sind. //

Doris Schulz, Fachjournalistin, Stuttgart 